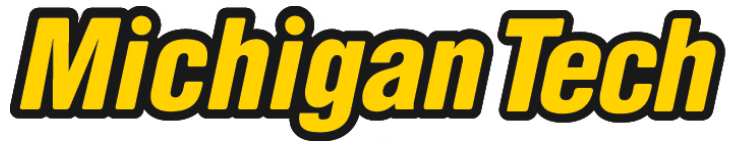 \\ Michigan Technological University Create the Future Digital Commons @ Michigan Tech
}

Dissertations, Master's Theses and Master's Reports - Open

Dissertations, Master's Theses and Master's

Reports

2011

Characterizing a single photon detector

Paul N. Rojas

Michigan Technological University

Follow this and additional works at: https://digitalcommons.mtu.edu/etds

Part of the Physics Commons

Copyright 2011 Paul N. Rojas

\section{Recommended Citation}

Rojas, Paul N., "Characterizing a single photon detector", Master's report, Michigan Technological University, 2011.

https://doi.org/10.37099/mtu.dc.etds/566

Follow this and additional works at: https://digitalcommons.mtu.edu/etds

Part of the Physics Commons 
CHARACTERIZING A SINGLE PHOTON DETECTOR

By

Paul N. Rojas

\begin{abstract}
A REPORT
Submitted in partial fulfillment of the requirements for the degree of MASTER OF SCIENCE PHYSICS
\end{abstract} MICHIGAN TECHNOLOGICAL UNIVERSITY 2011

(C) 2011 Paul Rojas 


\title{
Characterizing a Single Photon Detector
}

Paul Rojas

\author{
Advisor: Dr. Kim Fook Lee
}

\begin{abstract}
I will present my work about constructing and characterizing a single photon detector. Using the 1550nm laser and second harmonic light generation, I am able to count single photons on a Multi-Pixel Photon Counter (MPPC) silicon APD. My results show that upwards of $22 \%$ quantum efficiency is achievable with the MPPC. Future work will include coincidence detection of correlated photon-pair.
\end{abstract}

\section{Introduction}

\subsection{Motivation}

Quantum information is a big and growing field. One of the many topics includes quantum communication. Quantum communication utilizes photons as bits and by counting individual photons we take one step closer to realizing this goal. The wavelengths used in this experiment are also useful because they are in the telecom band. Another possible use is high resolution spectroscopy. With high resolution spectroscopy, individual photon resolution allows for more detailed spectrums, allowing for better characterization of elements.

\subsection{The Avalanche Photodiode}

The most important part of the experiment is the avalanche photodiode (APD). By use of an APD, which amplifies the effect of the photon, one can record measurements. An incident photon strikes an electron on the surface of the APD, with a large bias voltage greater than the breakdown voltage of the material, the electron speeds through the material, knocking more electrons thus creating the "avalanche". We use a power supply to create a bias voltage almost at the breakdown level, and then use a pulser with a voltage large enough such that the total voltage is above the breakdown voltage. There are two kinds of counts one can get from an APD, dark counts and light counts. Dark counts are when one sees the breakdown with no laser light. Light counts are counts from the APD while laser light is present. 


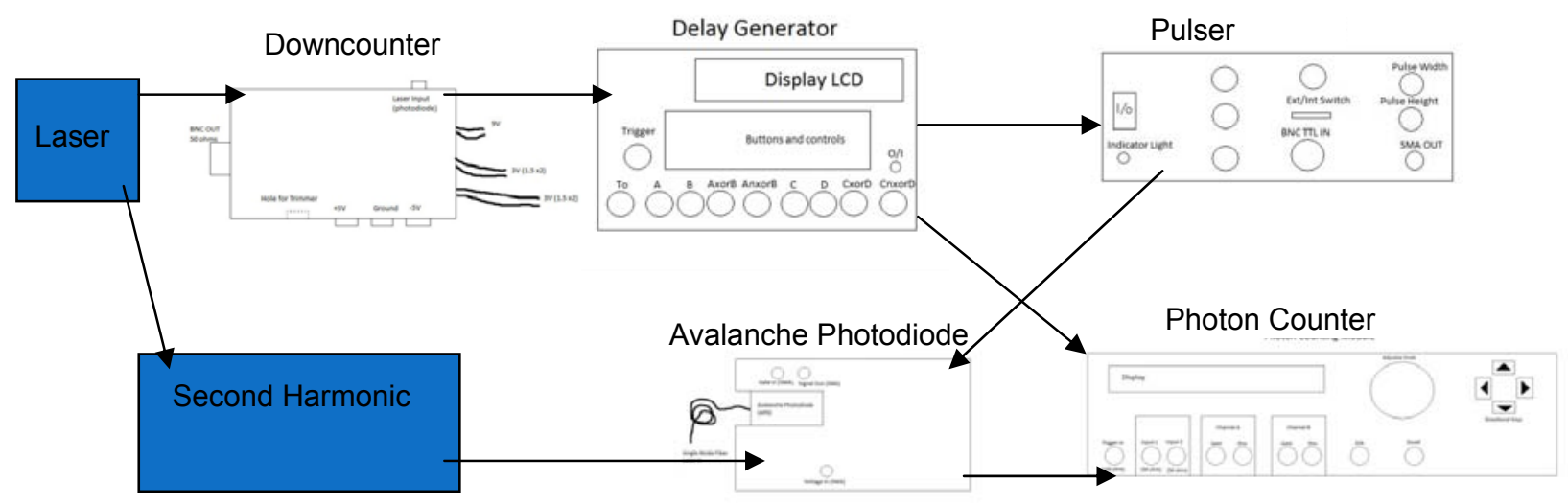

Figure 1. Diagram of Setup

\section{Components and Apparatus}

\subsection{Laser}

We use a $50 \mathrm{MHz}, 50 \mathrm{~mW}, 150 \mathrm{fs}$ pulse width, $1550 \mathrm{~nm}$ Polar Onyx laser for this operation. It is split into two beams, one at $49 \mathrm{~mW}$ and another at $1 \mathrm{~mW}$. The $1 \mathrm{~mW}$ beam is used as the trigger for the experiment; this beam goes to the downcounter. Unfortunately, $50 \mathrm{MHz}$ is too fast for our delay generator, which has a limit of $1 \mathrm{MHz}$, so we've slowed it down to $780 \mathrm{kHz}$. The $49 \mathrm{~mW}$ component is sent to the second harmonic light generation setup which allows us to halve the wavelength. This is necessary since our APD won't operate at an optical wavelength of $1550 \mathrm{~nm}$, however, $775 \mathrm{~nm}$ is within the APD's operating limits.

\subsection{Downcounter}

The downcounter inputs the laser and slows it down to $1 / 64^{\text {th }}$ of what its original frequency was. We must slow it down as the original $50 \mathrm{MHz}$ is too fast for our delay generator. The downcount is achieved by use of 4 components: the photodiode, an amplifier, a comparator, and a downcouter chip. The photodiode translates the optical signal into a digital signal, allowing us to use electrical components. Next, the electronic signal is sent to an amplifier, this brings the peak to peak voltage level of the signal into operating range of the comparator. Following the amplifier the signal goes to the comparator and is compared to a tunable resistor, resulting in a square wave. The square wave then goes to the downcounter chip and is downcounted by 64 . With an input frequency of $50 \mathrm{MHz}$ this results in a downcounted output frequency of $780 \mathrm{kHz}$. 


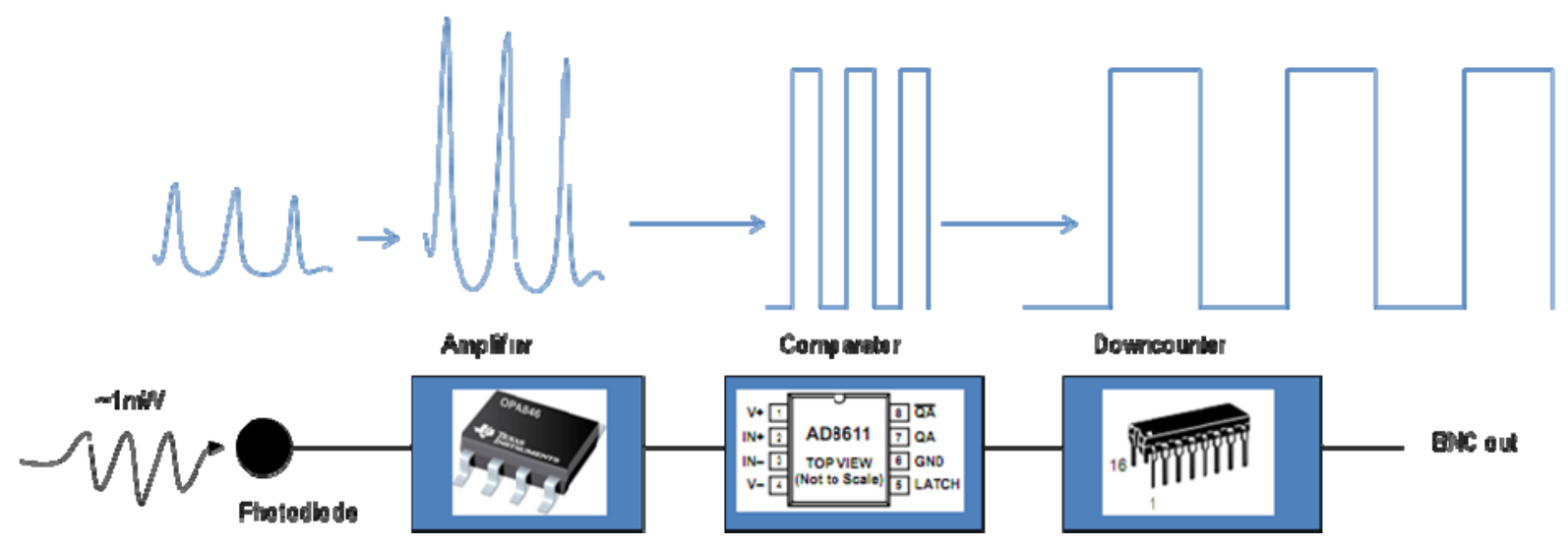

Figure 2. Diagram of the function of the downcounter circuit (See appendix A for full electronic diagram). First the photodiode takes the incident light and creates a signal. That signal goes to the amplifier, creating a gain. The comparator compares the signal with a resistor resulting in a square wave. This goes to the downcounter which slows the signal.

\subsection{Delay Generator}

Using the output from the downcounter we trigger the delay generator, an SRS DG535. The main function of the delay generator is to create multiple outputs that are synced up so our signals arrive at expected times. The delay generator is also able to create variable delays in its outputs. The maximum delay time for a signal is $1 / f-1 \mu \mathrm{s}$, where $f$ is the frequency. This places a maximum frequency of the device at $1 \mathrm{MHz}$. We output two TTL pulses, one to trigger our pulser and another to trigger our photon counter.

\subsection{Pulser}

Our pulse generator is an Avtec AVM-2-C. We can get pulses from $600 \mathrm{mV}$ to $15 \mathrm{~V}$ with widths of $500 \mathrm{ps}$ to $1.5 \mathrm{~ns}$. The pulser was $1.5 \mathrm{~ns}$ wide and $600 \mathrm{mV}$ high. It is important for us to be able to use small pulse widths because this means the APD will be above the breakdown voltage for a shorter amount of time resulting in less dark counts. Dark counts are counts made by the equipment when there is no laser light present.

\subsection{APD}

The APD we are calibrating is a silicon Multi-Pixel Photon Counter (MPPC) from Hamamatsu. It operates in a spectrum of laser light from $500 \mathrm{~nm}$ to $900 \mathrm{~nm}$ and has a breakdown voltage of 71.59 volts. The APD gets a bias voltage of $71 \mathrm{~V}$ from an Agilent power supply and gated voltage of $600 \mathrm{mV}$ from the pulser, which results in a total voltage of $71.6 \mathrm{~V}$, just above the breakdown voltage. The MPPC has a surface of pixels, this is where the incident laser light that is counted strikes. The signal from the MPPC is then sent to Input 1 on the photon counter for discriminated counting. 

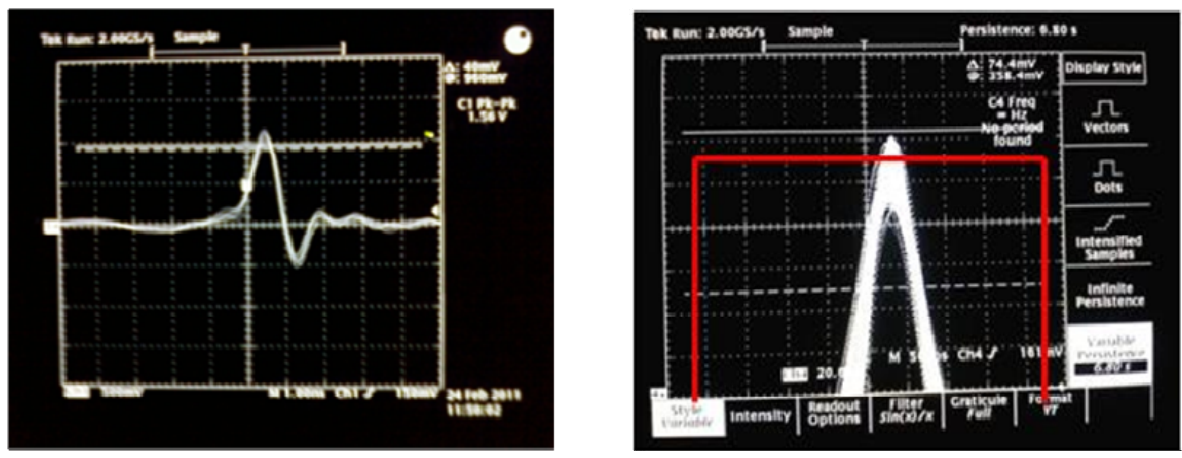

Figure 3. The left graph is shows a peak from our pulser. The right graph shows our breakdown signal outputted from our MPPC. Note that the signal becomes "sketchy" resulting from dark counts. The red overlay signifies what our discriminator from the photon counter might look compared to the signal.

\subsection{Second harmonic light generation}

Second harmonic light generation is a non-linear optical property of certain materials. Essentially, by sending a laser to a special kind of crystal, two photons will enter, but one will leave. This results in a doubling of energy thus a doubling of frequency and a halving of wavelength. This is necessary for our experiment because our MPPC APD operates at $775 \mathrm{~nm}$, which is approximately half of our 1550nm wavelength from our laser.

For the second harmonic light generation in this experiment, we used a Type I BBO crystal with a maximum efficiency of $\sim 2 \%$. To filter out the $1550 \mathrm{~nm}$ wavelength we used a broca prism. The broca prism has an index of refraction dependant on frequency which splits the laser light into $1550 \mathrm{~nm}$ and $775 \mathrm{~nm}$ beams. We block the $1550 \mathrm{~nm}$ light and couple the $775 \mathrm{~nm}$ light to a fiber optic cable. We were able to get a maximum power of $.9 \mathrm{~mW}$ from the $775 \mathrm{~nm}$ light, which is just under the $2 \%$ maximum efficiency, .98mW

Because we are characterizing the system, we must send a single photon per pulse of laser light to the APD. We achieve this by attenuating the light. By using the following equation: $P=R^{*} h^{*} c / \lambda$, where $R$ is the repetition rate of the laser, $h$ is Planck's constant, $c$ is the speed of light, and $\lambda$ is the wavelength of laser light, we calculate a power of $-79 \mathrm{dBm}$. Once the laser was attenuated to the proper power, we send that laser light to the APD.

\subsection{Photon Counter}

The photon counter we use is the SRS400. The counter accepts the TTL pulse from the delay generator. This machine runs for a specified number of gates, adjustable by the user, and marks a count whenever the input signal jumps above the discriminator level. The machine also gates our signal, thus allowing us to use only the biggest peak, where the breakdown is most significant.

\subsection{Problems}

We encountered multiple problems while building and running this experiment. Our first APD broke so we had to replace it with a cheaper APD. We also had issues with grounding, where getting physically 
close to wires and certain components caused our data to change. We also encountered a problem where our signal would drift on the photon counter, thus requiring us to constantly change the discriminator level to match where the new maximum would be.

Initially we used a Princeton Lightwave APD (PGA-400), with a pigtail coupling for light. This broke under use and we had to switch to the Hamamatsu APD, a cheaper, lower quality APD. We used the MPPC to test the circuit and find out what fried the PGA-400. The day it died there was a thunderstorm which could have created a power surge in the Agilent power supply and brought the total bias voltage too high for the APD. We don't believe this is case though due to the high quality of Agilent's power supplies, but it is still a possible factor though. Another problem we noticed was with our APD circuit, we had a dead capacitor. With a dead capacitor more DC voltage could have gotten to the APD thus killing it. However, we believe the main cause of the failure to be a short in the board. Due to using surface mount components and a small board its possible some solder created a bridge and allowed more voltage than necessary.

To fix the board we re-soldered the whole thing, once again taking care to how we soldered, and replaced the dead capacitor. Replacing the dead capacitor fixed our drifting problem and we remained with a constant steady signal. To fix our grounding issues we placed the APD into a metal box which relieved most of the sensitivity of the equipment.

\section{Results and Analysis}

To characterize the APD we recorded the number of counts per $10 \mathrm{k}$ gates. We checked to see how counts were affected by discriminator levels and bias voltages. Starting at the $17.8 \mathrm{mV}$ level for our discriminator and decreasing $.2 \mathrm{mV}$ each trial until $17.2 \mathrm{mV}$ since at that level there was an incredibly high dark count. To check the effect of bias voltage, we raised the total voltage to $71.8 \mathrm{~V}$ in $.1 \mathrm{~V}$ increments from 71.6V. For our errors we used the standard deviation for a Poisson system, $\sigma=(\mathrm{N})^{.5}$.

(a)

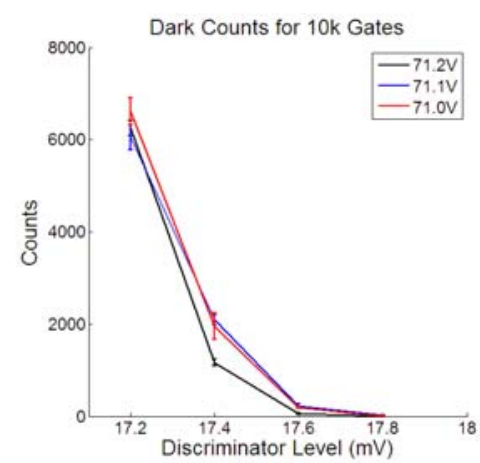

(b)

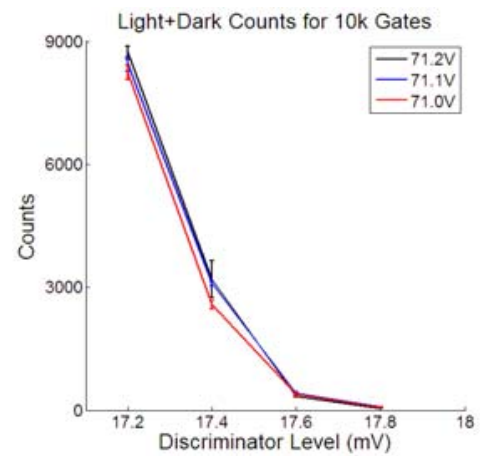

(c)

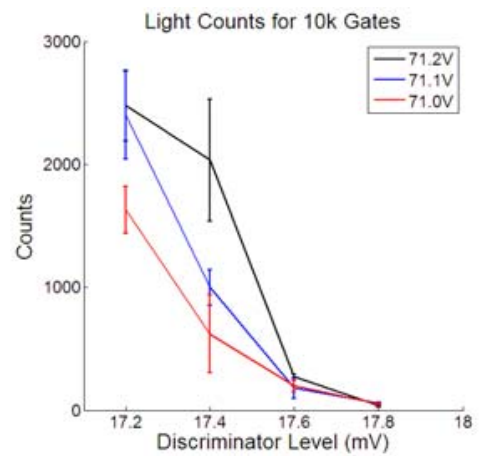

We took our measurements without light to get our dark count graphs. To get the light + dark count graph we took measurements with laser light incident to the APD. To find out the number of light counts though, one must subtract the dark counts from the light dark counts. Our results here are expected and make sense. By decreasing the discriminator level we increase the number of count. This is correct because as we lower the discriminator we lessen the requirement of energy needed to mark 
as a count. Also to note, for the light counts we see increasing counts as the bias voltage is raised. Once again, we expect this, because a greater bias voltage creates a greater avalanche effect on the diode making a bigger signal. On graph (c) the $71.2 \mathrm{~V}$ graph doesn't increase as much as one would expect compared to the lower bias voltages. This is because at a low discriminator level we have a high number of dark counts.

In an attempt to lower our error and bring our gates into a larger time domain, we combined our counts from the $10 \mathrm{k}$ gates and created data for $40 \mathrm{k}$ gates. For $40 \mathrm{k}$ gates we see the same trends as before.

(d)

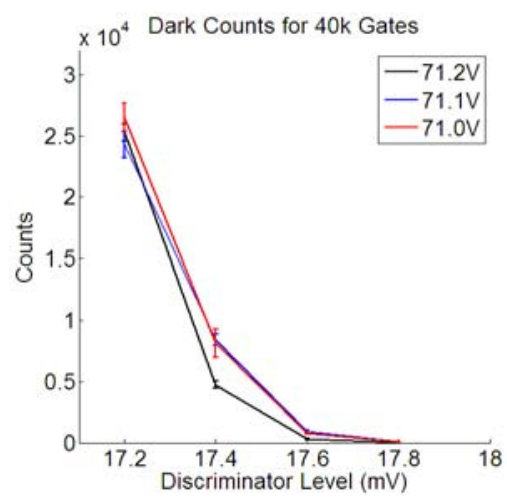

(e)

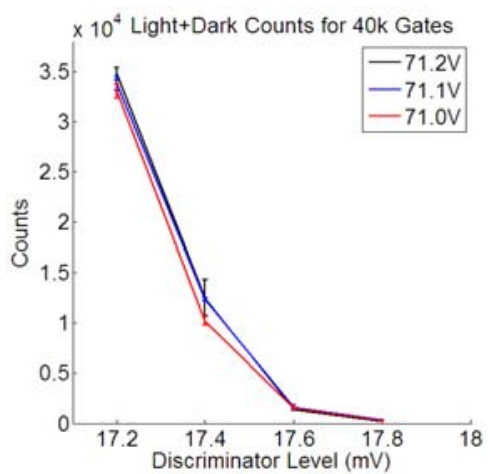

(f)

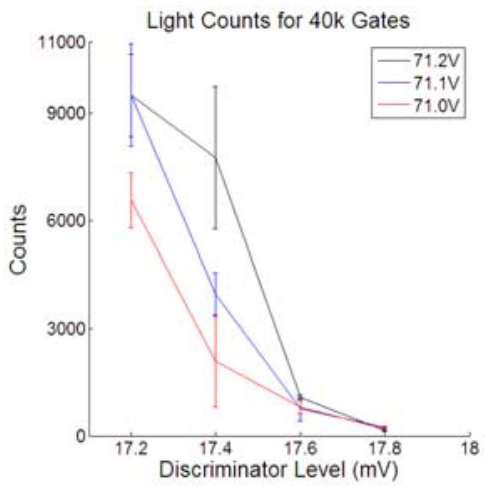

To characterize our MPPC silicon APD, we look at how quantum efficiency relates to the dark count probability and discrimination level. The dark count probability is simply the number of dark counts divided by the number of gates. For our experiment, we used one photon per pulse. This means our quantum efficiency is defined as:

\section{$\mathrm{QE}=$ number of light counts/number of gates}

In this case we use one photon per pulse; this means our efficiency is equal to the light count probability.

(g)

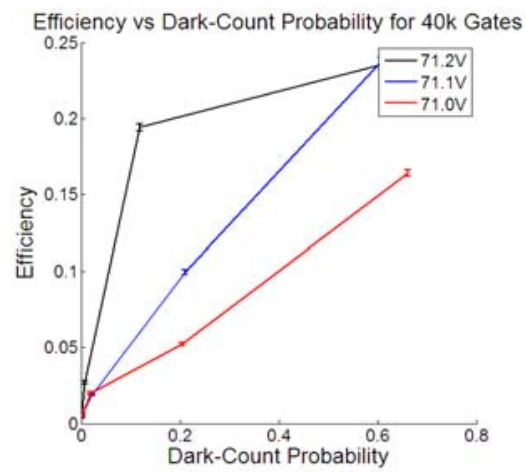

(h)

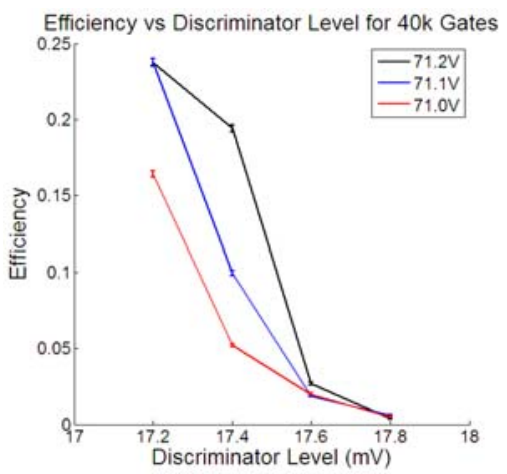


This shows our MPPC APD is able to achieve upwards of $22 \%$. However, due to the quality of the MPPC we have a high dark count probability. In graph (h) the efficiency increases as the discriminator is decreased due to increased light count probability.

\section{Future Work}

We are confident that our experiment works. Next, we must replace the Hamamatsu MPPC with our Princeton Lightwave APD and confirm that our setup still works it. By replacing it with an InGaAs APD we should keep the same efficiency but have a lower dark count probability, resulting in a better detector. Also, by using the InGaAs APD we will be able to get rid of the second harmonic light generation component because the InGaAs operates at $1550 \mathrm{~nm}$ wavelengths.

After we characterize our Princeton Lightwave InGaAs APD we will build a second APD circuit with the same type of APD in it and begin correlated photon counting. The SRS400 Photon Counter has two

inputs, allowing us to use two InGaAs APDs. We can then output the counts to our SR430 Multichannel Scaler and Averager; this will allow for coincidence counting. 


\section{References:}

- $14 M h z$ rate photon counting with room temperature InGaAs/InP avalanche photodiodes. Paul Voss et. al. Journal of Modern Optics. July 15 Vol. 51, n.9, 1369-1379.

- A Single-Photon Detector for High-Speed Telecom-Band Quantum Communication Applications. Chuang Liang et. al. Quantum Communications and Quantum Imaging III, Proc. of SPIE Vol. 5893. pg. 14-1 to $14-6$ 


\section{Appendix}

The electronic schematic for our downcounter circuit.

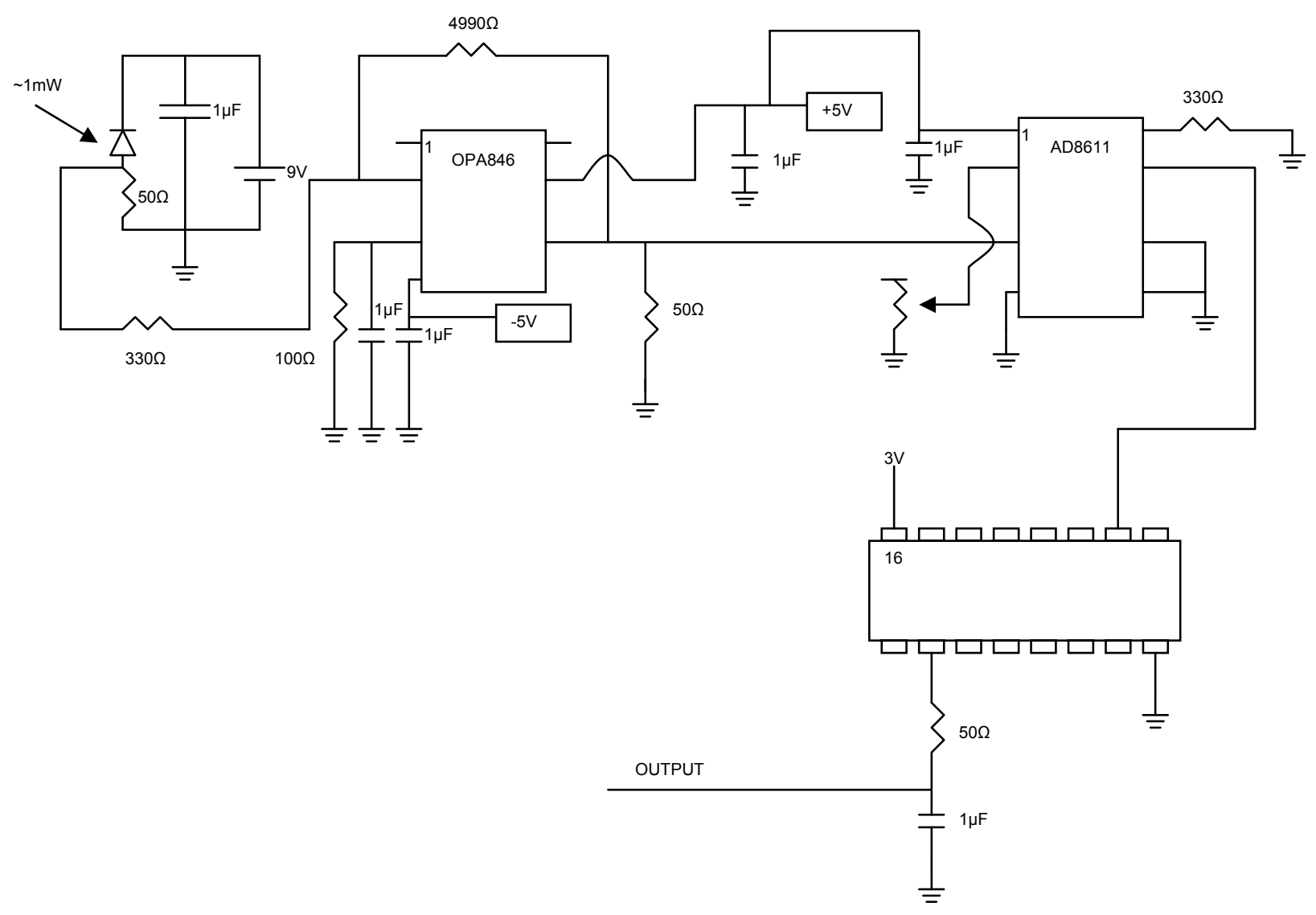


Electronic diagram for APD board

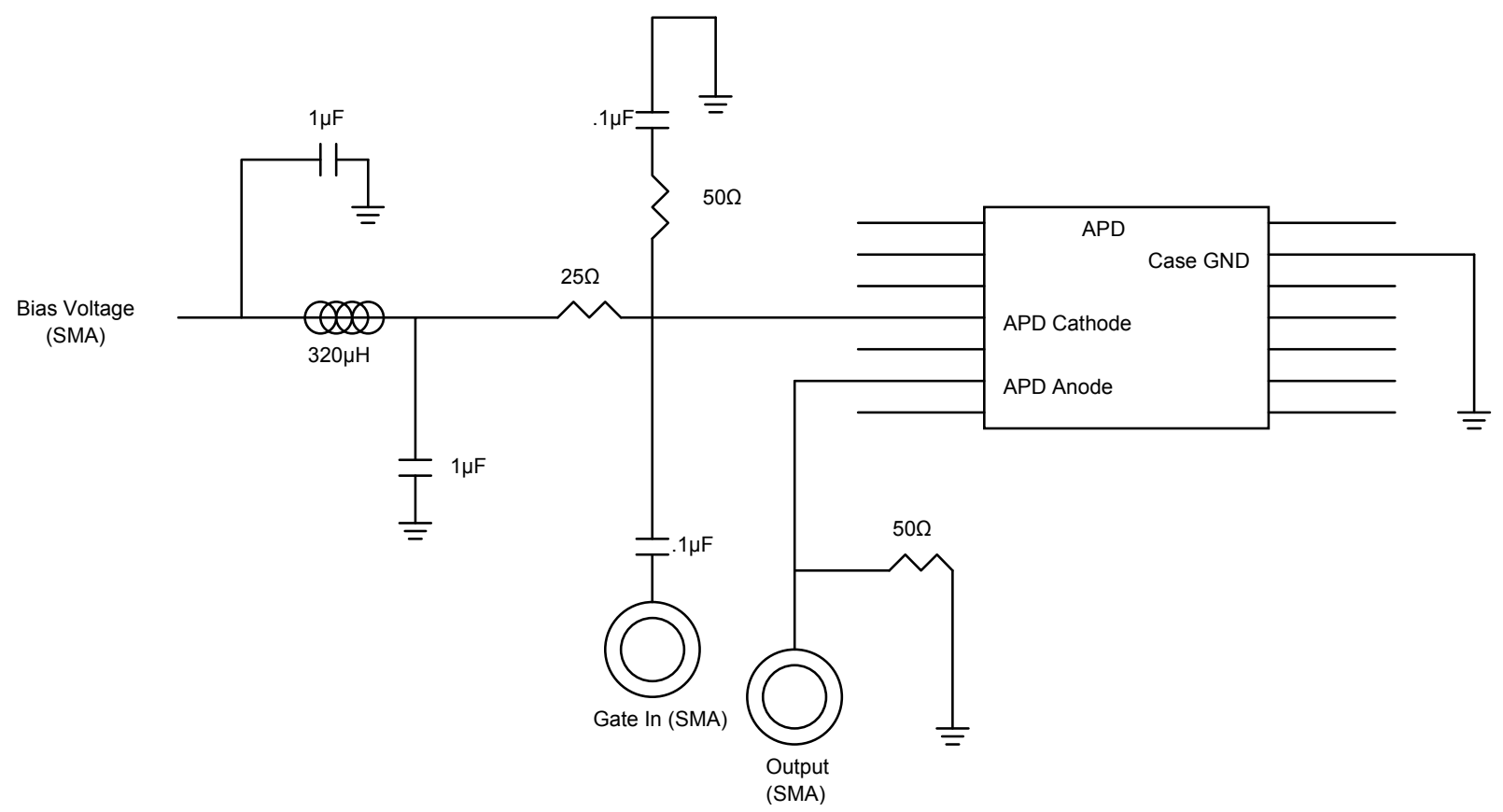

